\title{
FURTHER DIVISIBILITY PROPERTIES OF THE $q$-TANGENT NUMBERS
}

\author{
DOMINIQUE FOATA
}

\begin{abstract}
The $q$-tangent number $T_{2 n+1}(q)$ is shown to be divisible by $(1+q)^{a(n, 1)}\left(1+q^{2}\right)^{a(n, 2)} \ldots\left(1+q^{n}\right)^{a(n, n)}$, where the $a(n, i)$ 's are positive integers having the maximal property that $a(n, 1)+a(n, 2)+\cdots+a(n, n)=2 n$ whenever $n$ is a power of 2 .
\end{abstract}

1. Introduction. The $q$-tangent numbers are polynomials that may be defined by

$$
\begin{aligned}
& \sum_{n>0} T_{2 n+1}(q) x^{2 n+1} /(q ; q)_{2 n+1} \\
&=\left(\sum_{n}(-1)^{n} x^{2 n+1} /(q ; q)_{2 n+1}\right) /\left(\sum_{n>0}(-1)^{n} x^{2 n} /(q ; q)_{2 n}\right),
\end{aligned}
$$

where $(a ; q)_{n}=(1-a q) \ldots\left(1-a q^{n-1}\right)$ for $n>1$ and $(a ; q)_{0}=1$. When $q$ equals 1 , the $q$-tangent numbers become the ordinary tangent numbers $T_{2 n+1}$ $(n \geq 0)$ occurring in the Taylor expansion of $\tan x$

$$
\sum_{n>0} T_{2 n+1} x^{2 n+1} /(2 n+1) !=\tan x .
$$

Because of the relation

$$
(n+1) T_{2 n+1}=2^{2 n} G_{2 n+2},
$$

where $G_{2 n+2}$ is an odd integer called the Genocchi number (see e.g. [3]), Schützenberger [6] raised the problem of finding a polynomial of the form

$$
\prod_{i>1}\left(1+q^{i}\right)^{a(n, i)}
$$

that divides $T_{2 n+1}(q)$. Along these lines Andrews and Gessel [2] proved that $T_{2 n+1}(q)$ is divisible by

$$
A G_{n}(q)=(1+q)^{[n / 2]+1}\left(1+q^{2}\right) \ldots\left(1+q^{n}\right) .
$$

The purpose of this paper is to extend the result of the latter work as follows.

Every integer $n$ may be written as $n=m 2^{l}$ with $m$ odd and $l>0$, so that the polynomial

$$
E v_{n}(q)=\prod_{0<j<l}\left(1+q^{m 2^{j}}\right)
$$

Received by the editors October 25, 1979.

AMS (MOS) subject classifications (1970). Primary 05A15, 05A19; Secondary 33A30. 
may be defined (and is always divisible by $\left(1+q^{n}\right)$ ). Let

$$
\begin{aligned}
D_{n}(q) & =\prod_{1<i<n} E v_{i}(q) \quad \text { for } n \text { odd, } \\
& =\left(1+q^{2}\right) \prod_{1<i<n} E v_{i}(q) \quad \text { for } n \text { even, }
\end{aligned}
$$

or, in an equivalent manner,

$$
D_{1}(q)=1+q, \quad D_{2}(q)=(1+q)^{2}\left(1+q^{2}\right)^{2}
$$

and for $n \geqslant 3$

$$
D_{n}(q)=D_{n-2}(q) E v_{n-1}(q) E v_{n}(q) .
$$

For convenience, let $E v_{0}(q)=D_{0}(q)=1$.

THEOREM 1. For each $n \geqslant 0$ the polynomial $D_{n}(q)$ is a divisor of $T_{2 n+1}(q)$.

As $(1+q)\left(1+q^{n-1}\right)\left(1+q^{n}\right)$ divides $E v_{n-1}(q) E v_{n}(q)$ for every $n>2$, formula (1.8) shows by induction that $A G_{n}(q)$ (given in (1.4)) divides $D_{n}(q)$ for all $n$. Thus Theorem 1 extends the result obtained by Andrews and Gessel. Now to compare the divisibility properties of $A G_{n}(q)$ with $D_{n}(q)$ write the latter polynomial in the form

\begin{tabular}{|c|c|c|c|c|c|c|c|c|}
\hline$n$ & 1 & 2 & 3 & 4 & 5 & 6 & 7 & 8 \\
\hline 1 & 1 & & & & & & & \\
\hline 2 & 2 & 2 & & & & & & \\
\hline 3 & 2 & 1 & 1 & & & & & \\
\hline 4 & 3 & 3 & 1 & 1 & & & & \\
\hline 5 & 3 & 2 & 1 & 1 & 1 & & & \\
\hline 6 & 3 & 3 & 2 & 1 & 1 & 1 & & \\
\hline 7 & 3 & 2 & 2 & 1 & 1 & 1 & 1 & \\
\hline 8 & 4 & 4 & 2 & 2 & 1 & 1 & 1 & 1 \\
\hline
\end{tabular}

$$
D_{n}(q)=\prod_{1<i<n}\left(1+q^{i}\right)^{a(n, i)} .
$$

The first values of the coefficients $a(n, i)(1<i<n)$ are shown in Table 1 .

TABle 1

The number of factors in $A G_{n}(q)$ (resp. $D_{n}(q)$ ) is $[n / 2]+n$ (resp. $a(n, 1)$ $+\cdots+a(n, n))$.

Proposition 2. If $2^{l}<n<2^{l+1}(l>1)$, then

$$
(a(n, 1)+\cdots+a(n, n))-([n / 2]+n)>2^{l-1}-1 \text {. }
$$

Finally, as $D_{n}(1)$ divides the tangent number $T_{2 n+1}$, it follows from (1.9) and (1.3), since $G_{2 n+2}$ is odd, that

$$
a(n, 1)+\cdots+a(n, n)<2 n .
$$


Whenever equality holds, we may say that $D_{n}(q)$ is maximal.

Proposition 3. When $n$ is a power of 2 , then

$$
a(n, 1)+\cdots+a(n, n)=2 n,
$$

i.e. $D_{n}(q)$ is maximal.

The pattern of the paper by Andrews and Gessel is followed closely. In particular, the crucial part is played by a divisibility property of Gaussian polynomials (Lemma 2.2) that, roughly speaking, sorts the cyclotomic polynomials $\phi_{d}$ according to the parity of $d$.

2. A divisibility property of Gaussian polynomials. The polynomials $E v_{n}(q)$ defined in (1.5) can be expressed in terms of cyclotomic polynomials $\phi_{d}$ as follows.

LEMMA 2.1. For each $n \geqslant 1$ we have

$$
E v_{n}(q)=\prod\left\{\phi_{d}(q): d \mid 2 n, d \text { even }\right\} .
$$

Proof. Let $n=m 2^{l}$ with $m$ odd and $l>0$. For each $j=0,1, \ldots, l$ consider the set

$$
A_{j}=\left\{d: d \mid m 2^{j+1}, d \nmid m 2^{j}\right\}
$$

and let

$$
B=\left\{d: d \mid m 2^{l+1}, d \text { even }\right\}
$$

By definition

$$
1-q^{i}=\prod_{d \mid i} \phi_{d}(q) \text { for each } i>1 .
$$

As $\left(1-q^{2 i}\right)=\left(1-q^{i}\right)\left(1+q^{i}\right)$, we then derive

$$
1+q^{i}=\prod\left\{\phi_{d}(q): d \mid 2 i, d \nmid i\right\} .
$$

In particular, if $0 \leqslant j \leqslant l$, then

$$
1+q^{m 2^{j}}=\prod\left\{\phi_{d}(q): d \mid m 2^{j+1}, d \nmid m 2^{j}\right\}=\prod_{d \in A_{j}} \phi_{d}(q)
$$

On the other hand, as the sets $A_{j}$ are two by two disjoint, it suffices to show that $B$ is the union of the $A_{j}$ 's.

But if $d \mid m 2^{j+1}, d \nmid m 2^{j}$ for some $j$ with $0<j<l$, then $d \mid 2 n$ (equal to $m 2^{l+1}$ ) and $d$ is even. Thus $d$ belongs to $B$. Conversely, suppose $d \mid 2 n$ and $d$ even. Then $d=m^{\prime} 2^{j+1}$ with $m^{\prime}$ odd, $m^{\prime} \mid m$ and $0 \leqslant j<l$. Consequently, $d$ is an element of $A_{j}$. Q.E.D.

For each $n \geqslant 1$ let

$$
\operatorname{Od}_{n}(q)=\prod\left\{\phi_{d}(q): d \mid 2 n, d \text { odd }\right\}
$$

so that

$$
1-q^{2 n}=O d_{n}(q) E v_{n}(q)
$$


On the other hand, let the Gaussian polynomial be defined by

$$
\begin{aligned}
{\left[\begin{array}{l}
N \\
M
\end{array}\right] } & =(q ; q)_{N} /\left((q ; q)_{M}(q ; q)_{N-M}\right) \text { for } 0<M<N, \\
& =0 \text { otherwise. }
\end{aligned}
$$

LEMMA 2.2. For nonnegative integers $k$ and $n$ the expression

$$
\left[\begin{array}{c}
2 n \\
2 k+1
\end{array}\right] \frac{E v_{0}(q) E v_{1}(q) \ldots E v_{k}(q)}{E v_{n-k}(q) E v_{n-k+1}(q) \ldots E v_{n}(q)}
$$

is a polynomial in $q$.

Proof. The expression (2.6) is zero if $k>n$. Assume that $0<k<n-1$. Using (2.5) the Gaussian polynomial $\left[\begin{array}{c}2 n \\ 2 k+1\end{array}\right]$ may be factorized as a product of two factors,

$$
\begin{aligned}
{\left[\begin{array}{c}
2 n \\
2 k+1
\end{array}\right]=} & \frac{\operatorname{Od}_{n}(q)\left(1-q^{2 n-1}\right) O d_{n-1}(q) \ldots\left(1-q^{2 n-2 k+1}\right) O d_{n-k}(q)}{\left(1-q^{2 k+1}\right) O d_{k}(q)\left(1-q^{2 k-1}\right) \ldots O d_{1}(q)(1-q)} \\
& \cdot \frac{E v_{n}(q) E v_{n-1}(q) \ldots E v_{n-k}(q)}{E v_{k}(q) E v_{k-1}(q) \ldots E v_{1}(q)}
\end{aligned}
$$

When numerators and denominators are expressed in terms of cyclotomic polynomials, the first factor, because of (2.2) and (2.4) (resp. the second factor, because of (2.1)) only involves cyclotomic polynomials $\phi_{d}$ with $d$ odd (resp. $d$ even). As $\left[\begin{array}{c}2 n \\ 2 k+1\end{array}\right]$ is a polynomial and the cyclotomic polynomials are irreducible, each of these two factors is also a polynomial. But the first one is precisely equal to the expression given in (2.6). Q.E.D.

3. Proof of Theorem 1. Let $T(x)$ be the generating function for the $q$-tangent numbers as written in (1.1). Andrews and Gessel [2, p. 282] found that

$$
T(x)=(-i)\left((-i x ; q)_{\infty}-(i x ; q)_{\infty}\right) /\left((-i x ; q)_{\infty}+(i x ; q)_{\infty}\right)
$$

where $(a ; q)_{\infty}=\lim _{n}(a ; q)_{n}$. As $(a ; q)_{\infty}=(1-a)(a q ; q)_{\infty}$, it is straightforward to obtain

$$
T(x)-T(q x)=x+x T(q x) T(x)
$$

that is,

$$
\begin{aligned}
\sum_{n>0} T_{2 n+1}(q) x^{2 n} /(q ; q)_{2 n}= & 1+\left(\sum_{k>0} T_{2 k+1}(q) q^{2 k+1} x^{2 k+1} /(q ; q)_{2 k+1}\right) \\
& \cdot\left(\sum_{j>0} T_{2 j+1}(q) x^{2 j+1} /(q ; q)_{2 j+1}\right) .
\end{aligned}
$$

Equating coefficients of $x^{2 n}$ in both members we find that

$$
T_{2 n+1}(q)=\sum_{0<k<n-1}\left[\begin{array}{c}
2 n \\
2 k+1
\end{array}\right] q^{2 k+1} T_{2 k+1}(q) T_{2 n-2 k-1}(q) \quad(n>1) .
$$

The proof of Theorem 1 is now completed as follows. First $T_{1}(q)=T_{2 \times 0+1}(q)=$ 1. Proceed by induction on $n \geqslant 1$. For $0<k<n-1$ the expression

$$
\left[\begin{array}{c}
2 n \\
2 k+1
\end{array}\right] \frac{T_{2 k+1}(q) T_{2 n-2 k-1}(q)}{E v_{1}(q) \ldots E v_{n}(q)}
$$


is a polynomial because it may be factorized as

$$
\left[\begin{array}{c}
2 n \\
2 k+1
\end{array}\right] \frac{E v_{0}(q) \ldots E v_{k}(q)}{E v_{n-k}(q) \ldots E v_{n}(q)} \cdot \frac{T_{2 k+1}(q)}{E v_{0}(q) \ldots E v_{k}(q)} \cdot \frac{T_{2 n-2 k-1}(q)}{E v_{1}(q) \ldots E v_{n-k-1}(q)}
$$

the first factor being a polynomial by Lemma 2.2 ; the other two are also by the induction hypothesis. This shows, when $n$ is odd, that each term in the sum on the right side of $(3.3)$ is divisible by $D_{n}(q)=E v_{1}(q) E v_{2}(q) \ldots E v_{n}(q)$. Hence, $D_{n}(q)$ divides $T_{2 n+1}(q)$. When $n$ is even, rewrite (3.3) by grouping the terms two by two to give

$$
T_{2 n+1}(q)=\sum_{0<k<n / 2-1}\left[\begin{array}{c}
2 n \\
2 k+1
\end{array}\right] q^{2 k+1}\left(1+q^{2(n-2 k-1)}\right) T_{2 k+1}(q) T_{2 n-2 k-1}(q) .
$$

As $n$ is even, $\left(1+q^{2(n-2 k-1)}\right)$ is divisible by $\left(1+q^{2}\right)$, and by (3.4) the expression $\left[\begin{array}{c}2 n \\ 2 k+1\end{array}\right] T_{2 k+1}(q) T_{2 n-2 k-1}(q)$ is divisible by $E v_{1}(q) E v_{2}(q) \ldots E v_{n}(q)$. Hence, each term in the sum on the right side of (3.5) is divisible by $D_{n}(q)=$ $\left(1+q^{2}\right) E v_{1}(q) \ldots E v_{n}(q)$. This completes the proof of Theorem 1 .

4. Proofs of Propositions 2 and 3. Note that $D_{2}(q)=\left(1+q^{2}\right) E v_{1}(q) E v_{2}(q)=$ $(1+q)^{2}\left(1+q^{2}\right)^{2}$. Thus (1.12) holds for $n=2$. Let $n=2^{l}(l>2)$ and proceed by induction on $l$. Clearly

$$
E v_{2 i}(q)=E v_{i}(q)\left(1+q^{2 i}\right) \quad(i>1)
$$

Hence,

$$
\begin{aligned}
D_{n}(q) & =\left(1+q^{2}\right) \prod_{1<i<n} E v_{i}(q) \\
& =\left(1+q^{2}\right) \prod_{\substack{1<i<n \\
i \text { odd }}} E v_{i}(q) \cdot \prod_{\substack{1<i<n \\
i \text { even }}} E v_{i}(q) \\
& =\left(1+q^{2}\right) \prod_{1<i<n / 2}\left(1+q^{2 i-1}\right) \cdot \prod_{1<i<n / 2} E v_{2 i}(q) \\
& =\left(1+q^{2}\right) \prod_{1<i<n / 2}\left(1+q^{2 i-1}\right) \cdot \prod_{1<i<n / 2}\left(1+q^{2 i}\right) \cdot \prod_{1<i<n / 2} E v_{i}(q)
\end{aligned}
$$

By grouping the first and last factors we obtain

$$
D_{n}(q)=D_{n / 2}(q) \prod_{1<i<n}\left(1+q^{i}\right)
$$

Therefore, if the number of factors in $D_{n / 2}(q)$ is $n$, the polynomial $D_{n}(q)$ will have $n+n=2 n$ factors. This completes the proof of Proposition 3.

As for Proposition 2 let $d_{n}=a(n, 1)+\cdots+a(n, n)$ for $n>1$. From (1.6) and (1.9) it follows that $d_{2 n}=d_{2 n+1}$ for $n>1$. On the other hand, the number of factors in $A G_{2 n+1}(q)$ is $3 n+1$. Let $p=2^{l}<2 n<2^{l+1}(l>1)$. To prove Proposition 2 it suffices to show that $d_{2 n}-(3 n+1)>2^{l-1}-1$, i.e.

$$
d_{2 n}>3 n+2^{l-1} \text {. }
$$


From (1.8)

$$
D_{2 n}(q)=D_{p}(q) \prod_{p+1<i<2 n} E v_{i}(q)
$$

But, when $i$ is even, the polynomial $E v_{i}(q)$ is a product of at least two binomials $\left(1+q^{j}\right)$. Hence

$$
d_{2 n} \geqslant d_{p}+3(2 n-p) / 2=3 n+2^{l-1},
$$

which is inequality (4.2).

5. Concluding remarks. Recall that a permutation $x_{1} x_{2} \ldots x_{2 n+1}$ of the sequence $12 \ldots(2 n+1)$ is alternating if $x_{1}>x_{2}, x_{2}<x_{3}, \ldots, x_{2 n}<x_{2 n+1}$. As $[2 k+1]$ is the generating polynomial for permutations $x_{1} x_{2} \ldots x_{2 n}$ of $1^{2 k+1} 2^{2 n-2 k-1}$ by number of inversions (see e.g. [1, p. 41]), it is clear that the running term on the right side of (3.3) is the generating polynomial for alternating permutations $x_{1} x_{2} \ldots x_{2 n+1}$ of $12 \ldots(2 n+1)$ with $x_{2 k+2}=1$, by number of inversions, a result known to several authors [4]-[7]. The proof of Theorem 1 shows that the latter generating function is itself divisible by $E v_{1}(q) E v_{2}(q) \ldots E v_{n}(q)$. It would be interesting to have a combinatorial proof of this result by using that alternating permutation set-up.

From (3.1) Andrews and Gessel [2] derived the recurrence formula

$$
\begin{gathered}
T_{2 n+1}(q)+\sum_{1<j<n}(-q ; q)_{2 j-1}\left[\begin{array}{c}
2 n+1 \\
2 j
\end{array}\right](-1)^{j} T_{2 n+1-2 j}(q) \\
=(-1)^{n}(-q ; q)_{2 n} .
\end{gathered}
$$

It was not possible to use (5.1) directly to prove Theorem 1 because, for instance, when $n=2^{l}(l \geqslant 1)$ the polynomial $(-q ; q)_{2 n}$ is not divisible by $D_{n}(q)$. That is why we had to derive the quadratic recurrence formula (3.3).

\section{REFERENCES}

1. G. E. Andrews, The theory of partitions, Encyclopedia of Mathematics and its Applications, Vol. 2, Addison-Wesley, Reading, Mass., 1976.

2. G. E. Andrews and I. Gessel, Divisibility properties of the q-tangent numbers, Proc. Amer. Math. Soc. 68 (1978), 380-384.

3. D. Dumont, Interprétations combinatoires des nombres de Genocchi, Duke Math. J. 41 (1974), 305-318.

4. I. Gessel, Generating functions and enumeration of sequences, Ph. D. thesis, M.I.T., 1977.

5. D. Rawlings, Generalized Worpitzky identities, Univ. of California, San Diego, 1979 (preprint); European J. Combinatorics (to appear).

6. M. P. Schützenberger, Oral communication, Combinatorics Conference, Oberwolfach, 1975.

7. R. P. Stanley, Binomial posets, Möbius inversion and permutation enumeration, J. Combinatorial Theory Ser. A 20 (1976), 336-356.

Département de Mathématique, Université de Strasbourg, 7 Rue René Descartes, F 67084 Strasbourg, France 